\title{
EBUS-TBNA: Are Two Needle Revolutions (Back and Forth Movement of the Needle Inside the Lymph Node) Adequate for Diagnosis of Lung Cancer?
}

\author{
Akash Verma ${ }^{1 *}$, John Abisheganaden ${ }^{1}$, Poh Wee Teng ${ }^{1}$, Kent Mancer ${ }^{1}$ and Eric S. Edell ${ }^{2}$ \\ ${ }^{1}$ Department of Respiratory and Critical Care Medicine, Tan Tock Seng Hospital, Singapore \\ ${ }^{2}$ Division of Pulmonary and Critical Care Medicine, Mayo Clinic, USA
}

\begin{abstract}
Aim: The optimum number of revolutions (back and forth movement of the needle inside the lymph node) during endobronchial ultrasound guided transbronchial needle aspiration (EBUS-TBNA) remains to be determined. This study aimed to compare the performance of number of revolutions during EBUS-TBNA of mediastinal and hilar lymph nodes.

Methods: Prospective analysis of consecutive patients with mediastinal and hilar lymphadenopathy who underwent EBUS-TBNA over a 3-month period was done. Cytological or histological results from the specimen obtained using two revolutions were compared with 16 revolutions.

Results: Twenty four patients underwent sampling of 37 lymph node stations. No difference in the detection rate of the malignancy was noted between lymph node aspiration using 2 versus 16 revolutions $(p=0.058)$. This lack of difference was maintained whether the "worm like" string of core tissue was obtained or not $(p=0.08)$. Although the "worm like" string of core tissue was obtained significantly more frequently with 16 revolutions ( $p=0.0104)$, this did not influence the detection rate of malignancy. The average time taken from the start of the procedure to the completion of two revolutions was $11.8 \pm 5.6$ minutes.

Conclusion: More than two revolutions or "worm like" string of aspirate does not add value in diagnosing malignancy via EBUS-TBNA. In patients with high pre-test probability of lung cancer, adequacy of two revolutions may confer greater safety and efficiency to the procedure. However, in case of high suspicion of a benign disease, it may be preferable to seek "worm like" material to aid establishment of diagnosis.
\end{abstract}

Keywords: Endo bronchial ultrasound; Transbronchial needle aspiration; Passes; Revolutions; Bronchoscopy; Cancer (lung)

\section{Introduction}

EBUS-TBNA has revolutionized bronchoscopy adding significantly to the pre-existing utility of the flexible bronchoscopy since its development in 1960`s [1]. More and more institutes are adopting EBUS-TBNA globally because of its high diagnostic informative value and low risk. The sensitivity and rates of diagnostic accuracy of EBUSTBNA for differentiating malignant and benign disease has been reported from 85 to $93 \%$ and 88 to $91 \%$, respectively [2-5].

Despite a high diagnostic yield, the significant number of false negative findings in the cytology remains problematic. A numbers of studies examining epithelial malignancies have been conducted that highlight this predicament [6,7]. Additionally, the advent of targeted therapy and better outcomes in non-small cell lung cancer has made adequacy of tissue for histo-pathological sub-typing followed by mutation identification more important than before. It has been suggested that the generation of significant false negative results can be explained by detection error [7]. For example, the correct node is sampled but the material obtained does not represent the underlying disease. Several detection error related factors have been recognized and addressed such as; required number of aspiration per lymph node station (recommended three for diagnosis [8], and four for molecular analysis), needle gauge (21- and 22-gauge aspiration needles being found to be equally effective) $[9,10]$, suction pressure (high with large $30 \mathrm{ml}$ syringe better [11] versus no difference [12]), and the distance travelled by the needle within the lymph node (recommendation being that greater the distance moved by the needle, higher the yield).

The number of revolutions (number of times the needle should be moved back-and-forth inside the lymph node) is one such factor. The recommendation is to move ten to twenty times. However the optimum number of times the needle should be moved back and forth for extracting adequate tissue has not been studied to date. In addition to insufficient data in this area, the terminology like "number of passes"used in the literature to describe how many times any lymph node is punctured to obtain specimen is often confused with the back and forth movement of the needle [13-15]. This may be because, although "needle revolutions" (perhaps for the lack of a better word) has been used as the terminology to describe the back and forth movement of the needle inside the lymph node $[16,17]$ it is not routinely used in day to day conversation and teaching sessions.

In this study we focused on determining the adequate number of revolutions (back and forth movement of the needle in the lymph node) needed for establishment of the diagnosis.

\section{Methods and Procedures}

\section{Study design and patients}

Prospective analysis of consecutive patients with mediastinal and hilar lymphadenopathy who underwent EBUS-TBNA over a 3-month period ( $4^{\text {th }}$ November $2013-3^{\text {rd }}$ February 2014) was done. All patients

${ }^{*}$ Corresponding author: Akash Verma, MRCP, Tan Tock Seng Hospital, Department of Respiratory and Critical Care Medicine, 11 Jalan Tan Tock Seng, Singapore 308433, Tel: +65-63573790; Fax: +65-63573087; E-mail: Akash_Verma@ttsh.com.sg

Received November 07, 2014; Accepted January 26, 2015; Published January 30, 2015

Citation: Verma A, Abisheganaden J, Teng PW, Mancer K, Edell ES (2015) EBUSTBNA: Are Two Needle Revolutions (Back and Forth Movement of the Needle Inside the Lymph Node) Adequate for Diagnosis of Lung Cancer?. J Pulm Respir Med 5: 239. doi:10.4172/2161-105X.1000239

Copyright: $(2015$ Verma A, et al. This is an open-access article distributed under the terms of the Creative Commons Attribution License, which permits unrestricted use, distribution, and reproduction in any medium, provided the original author and source are credited. 
with enlarged mediastinal lymph nodes detected on the CT scan done to evaluate their symptoms or radiographic findings were included. EBUS-TBNA was done for both diagnosis and staging purpose as deemed necessary based on the clinical and radiological features along with degree of suspicion for malignancy. This study was approved by the institutional review board of the Changi General Hospital.

\section{Definitions}

We defined "passes" as the number of times the lymph node (LN) was punctured to obtain new/fresh sample of specimen. We defined "revolutions" as the number of times the needle was moved back and forth within the LN after puncturing. We defined the specimen obtained without worm like appearance as "aspirate" and the worm like material as "core tissue" Figure 1. The "aspirate" obtained was used to prepare a smear, and if a "core tissue" (worm like material) was obtained, it was collected separately in a formalin bottle.

\section{EBUS-TBNA procedure}

EBUS-TBNA was performed by two trained operators using a curvilinear scanning ultrasound bronchoscope (BF UC180F, Olympus Ltd., Tokyo, Japan) connected to an ultrasound unit (EU-ME1 Olympus Ltd., Tokyo, Japan). The procedures were performed under local anesthesia (lignocaine) and moderate sedation (midazolam). For paratracheal lesions, the scope was positioned endotracheally. For peribronchial lesions the scope was positioned in the respective bronchi. TBNA was performed using a 22-gauge needle (NA-201SX-4022, Olympus Ltd., Tokyo, Japan). A balloon was inflated around the tip of the scope in order to maintain contact with the airway wall. Once the target lymph node had been located (and vascular structures excluded with the Doppler function), the 22-G needle was placed in the working channel of the EBUS scope. The tip of the sheath of the needle was visible on the endobronchial view, and the needle was then allowed to pierce the airway wall and enter the lymph node using the jabbing technique under direct ultrasound guidance. Suction of $-15 \mathrm{~cm} \mathrm{H} 2 \mathrm{O}$ via dedicated vacuum syringe was applied and the needle moved to and fro within the lymph node. Sequential puncturing and aspiration was done of each LN station of interest with two revolutions, followed by 16 revolutions to compare the difference in the detection rate of diagnosis. With every revolution, needle was penetrated to pass beyond the mid line axis of the lymph node to ensure sampling from one end of the capsule to the other. While aspiration by two revolutions was only done once, the aspiration by 16 revolutions was done 2-3 times (depending on how much passes it took to obtain worm like material) with total number of passes per lymph node station being three. The core tissue was expelled onto a piece of paper for histological examination and the needle was flushed with saline onto glass slides for cytological examination. The aspirate was smeared onto glass slides, air dried, fixed immediately with $95 \%$ alcohol, and stained with Hematoxylin and Eosin (HE). The "worm like" histological cores were fixed with $10 \%$ neutral buffered formalin and stained with HE. Rapid on-site cytological examination was not available.

\section{Data analysis}

We used software (SPSS, version 17; SPSS, Chicago, Ill) for all statistical analyses. The cytological and histological results were compared using a Wilcoxon two-sample test. The adequacy of the samples obtained using 2 and 16 revolutions was compared using the McNemar's test. $P$ values were two sided and considered indicative of a significant difference if less than .05 .

\section{Results}

Thirty seven lymph node stations punctured in 24 patients were evaluated for the difference in diagnosis detection rate based on number of revolutions employed for aspiration of tissue. Out of 37, 29 were aspirated using 2 and 16 revolutions, 7 were aspirated using 16 revolutions alone, and 1 was aspirated using 2 revolutions alone. Clinical characteristics of patients are given in Table 1. Out of 37 stations, 25 yielded a diagnosis of either benign or malignant disease. Overall detection rate among all lymph node stations was 25/37 67.5\%. Out of 23 malignant stations, EBUS-TBNA revealed a diagnosis in 20 with the detection rate of $87 \%$. Out of 14 benign stations, EBUS revealed the diagnosis in 5 with the detection rate of $35.7 \%$. Overall EBUS-TBNA was significantly more diagnostic in malignant diseases as compared to benign $(\mathrm{p}=0.0028)$, Figure 2 . In six patients (12 lymph node stations) the EBUS-TBNA was negative. The final diagnosis in these patients was obtained by Nucleic acid amplification test positive for TB on pleural fluid $(n=1)$, Transbronchial needle flushings positive for tuberculosis (TB) $(n=2)$, mediastinoscopy positive for lymphoma $(\mathrm{n}=1)$, transthoracic needle aspiration (TTNA) positive for adenocarcinoma $(n=1)$, and pus aspiration from psoas muscle positive for tuberculosis $(n=1)$, Figure 3.

The analysis of the pathology results from the aspirate and core tissue combined, and aspirate alone, did not reveal significant difference in the detection rate of the malignant $(\mathrm{p}=0.058, \mathrm{p}=0.17)$ or benign diagnosis $(p=0.002, p=1.0)$ respectively between 2 versus 16 revolutions. This lack of difference was maintained whether the "worm like" string of core tissue was obtained or not. However significant difference in the detection rate of the malignant or benign diagnosis ( $\mathrm{p}=0.01, \mathrm{p}=0.042$ respectively) was noted when core tissue alone was analyzed likely secondary to obtaining worm like core tissue more frequently via 16 revolutions. Although the "worm like" string of core tissue was obtained significantly more frequently with 16 revolutions $(\mathrm{p}=0.0104)$ as compared to two, this did not influence the detection of malignancy Figure 4. However, none of the lymph node station with the benign disease yielded the diagnosis with two revolutions. All lymph node stations with benign disease only revealed diagnosis on "worm like" core tissue examination. The average time taken from the start of the procedure to the completion of 2 revolutions was $11.8 \pm 5.6$ minutes.

\section{Discussion}

In the current study we demonstrated that two needle revolutions were as adequate as sixteen, and the presence of "worm like material" on aspiration did not influence the detection rate of malignancy via EBUS-TBNA.

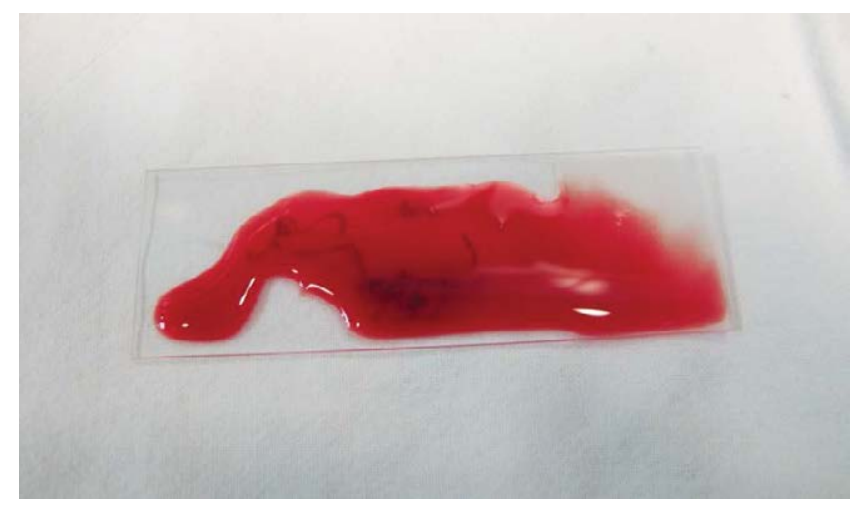

Figure 1: Worm like material obtained with 16 revolutions. 


\begin{tabular}{|c|c|}
\hline Variable & $\mathbf{N}$ (\%) or Median (range) \\
\hline Patients & 24 \\
\hline Age & $59(28-79)$ \\
\hline Gender & \\
\hline Male & $21(87.5)$ \\
\hline Lymph Node Stations (n=37) & $2(5)$ \\
\hline $2 R$ & $12(32)$ \\
\hline $4 R$ & $6(16)$ \\
\hline $4 L$ & $12(32)$ \\
\hline 7 & $2(5)$ \\
\hline $10 R$ & $1(2.7)$ \\
\hline $10 L$ & $1(2.7)$ \\
\hline $11 L$ & $1(2.7)$ \\
\hline $11 R$ & $2.1(1.1-5.4)$ \\
\hline Size of lymph node $\mathbf{( c m )}$ & \\
\hline Final diagnosis $\mathbf{( n = 3 7 )}$ & $23(62)$ \\
\hline Malignancy & $14(38)$ \\
\hline Benign & \\
\hline EBUS-TBNA diagnosis (n=25) & $20(80)$ \\
\hline Malignancy & $5(20)$ \\
\hline Benign & \\
\hline
\end{tabular}

EBUS-TBNA: Endobronchial ultrasound transbronchial needle aspiration Table 1: Clinical characteristics of the patients.

The sensitivity and rates of diagnostic accuracy of EBUS-TBNA for differentiating malignant and benign disease has been reported from 85 to $93 \%$ and 88 to $91 \%$, respectively [2-5]. In the current study we found that, overall EBUS-TBNA was diagnostic in 25/37 (67.5\%) of lymph node stations, and was significantly more diagnostic in malignant stations 20/23 (87\%) as compared to benign 5/14 (35.7\%). Although the overall detection rate of $67.5 \%$ is lower than the previous publications, this could be due to higher (38\%) prevalence of benign disease in our cohort (10 lymph node stations suspected of tuberculosis, 4 suspected of sarcoidosis). It has been reported that the rate of attainment of diagnostic material via TBNA is higher in malignancy as compared to benign disease $[18,19]$. Abu-Hijleh $\mathrm{M}$ et al reported EBUS-TBNA to be more accurate (96.12\% (CI 91.25-98.33)) with higher NPV (90.74 $\%$ (CI 80.09-95.98)) in patients with suspected malignancy compared with patients with suspected benign disease (79.31 \% (CI 67.23-87.75), $20 \%(7.05-45.19))$ in total of 690 TBNAs performed from $294 \mathrm{LN}$ stations [20]. Another reason for the overall low detection rate may be because we considered the pathological result of "atypical cells seen" as negative result.

Despite a high diagnostic yield, the significant number of false negative findings in the cytology remains problematic. A number of studies examining epithelial malignancies have been conducted that highlight this predicament $[6,7]$. It has been suggested that the generation of significant false negative results can be explained by detection error. Several detection error related factors have been recognized and studied such as; required number of aspiration per station $[8,13]$, needle gauge $[9,10]$, and suction pressure $[11,12]$. However, the optimal number of revolutions for extracting sufficient tissue sample during EBUS-TBNA has not been evaluated. The existing recommendation is to perform $10-20$ revolutions $[7,17,18]$. In the current study, no significant difference in the detection rate of the malignant or benign diagnosis was noted between lymph node aspiration using 2 versus 16 revolutions. In patients with malignancy, the results of aspirate and core tissue in combination was diagnostic in 14 and 19 stations via 2 and 16 revolutions respectively ( $\mathrm{p}=0.679$ ).
The aspirate alone was diagnostic in 12 and 15 stations via 2 and 16 revolutions $(\mathrm{p}=0.10)$. The core tissue alone was diagnostic in 5 and 16 stations via 2 and 16 revolutions respectively $(\mathrm{p}=0.01)$. When comparing the diagnosis detection rate via core tissue examination alone, a trend toward higher diagnostic rate was observed using 16 revolutions. This can be attributed to higher frequency of obtaining "worm like" core tissue by 16 revolutions. Similar trend was seen in benign diseases. The examination of core tissue alone showed a trend toward higher diagnostic rate with 16 revolutions. Although the results of aspirate and core tissue in combination showed higher diagnostic rate with 16 revolution, this was because in none of the benign cases did the aspirate yield the diagnosis. The lack of difference between 2 and 16 revolutions in unfolding the diagnosis can be conceivably explained by the movement of the needle along a fixed axis. When the needle first enters the lymph node following the puncture, and is advanced forward traversing the lymph node, the tissue gets filled in its lumen upon its first pass. It can be speculated that following this, if the angle of the needle and rotation of the scope is not changed, despite several back and forth movements, the needle is only going to move along the same path with each stroke, due to which not much additional tissue may enter into it in the incremental manner.

The detection error that has the potential of originating from inadequate revolutions is that, only cytology may be collected [7]. The experts in the technique of EBUS-TBNA teach that the macroscopic "worm like" material is good for histopathological analysis due to preservation of tissue architecture. The diagnostic yield has been reported to increase by extracting and sampling the "worm like" material as described by Koen et al. in their case report. These authors recommend using 10-15 passes as the lesser number of passes fail to give "worm like" material (core tissue) [7]. However some Bronchoscopists argue that 2 passes may suffice and what appears as core tissue in the form of worm like material may just be blood. In the current study we noted that the worm like material was indeed more often obtained with $>16$ revolutions, consistent with the findings of Koen et al, however, were only helpful in establishing the diagnosis of the benign disease. Its absence did not preclude the establishment of the diagnosis of malignancy.

The average procedure time of EBUS-TBNA is $45 \pm 13$ minutes [21]. This includes white light bronchoscope insertion for initial screening to EBUS scope removal from the airway. When we assessed the time interval between WLB insertions to completion of 2 passes from the first lymph node station, it was $11.8 \pm 5.6$ minutes. This

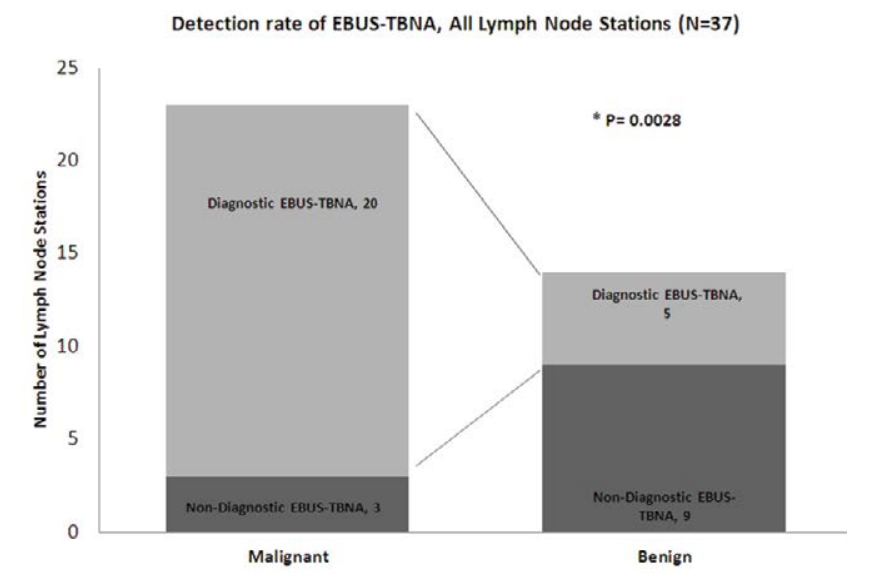

Figure 2: Overall EBUS-TBNA was significantly more diagnostic in malignant diseases as compared to benign $(P=0.0028)$ 


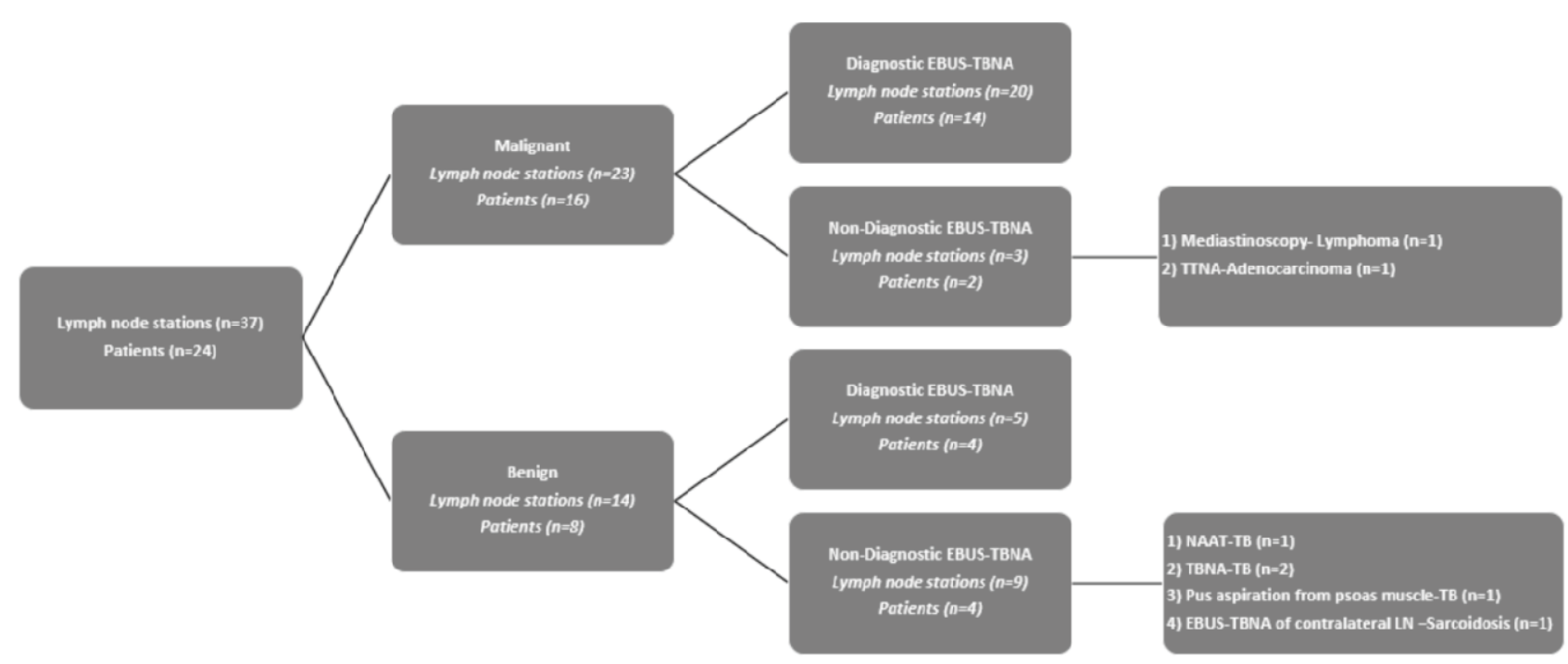

Figure 3: Consort flow diagram of patients and diagnosis.

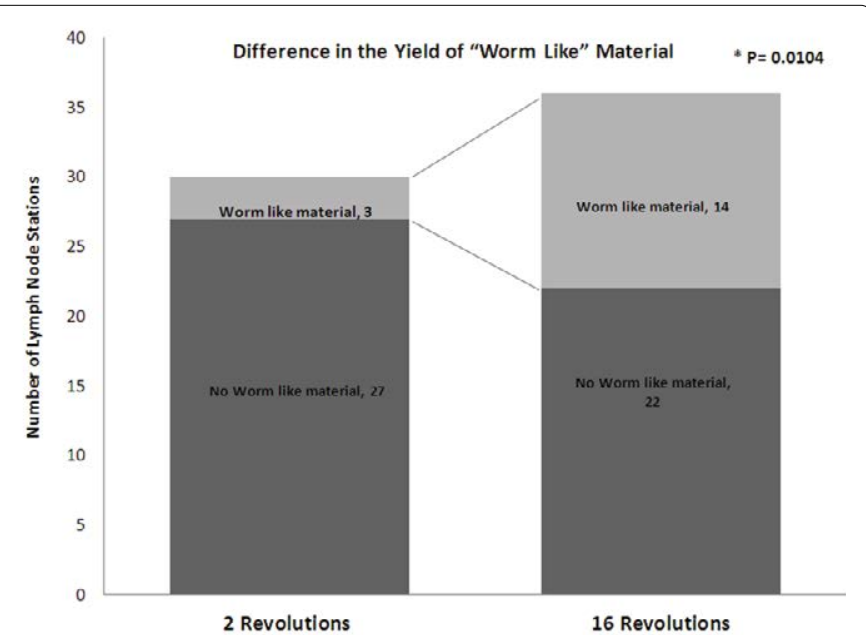

Figure 4: Although the worm like string of core tissue was obtained significantly more frequently with 16 revolutions $(P=0.0104)$ as compared to two, this did not influence the diagnosis detection rate in case of malignancy.

procedure time (scope in-scope out time) is much shorter and carries the potential for reduction in the total cases times. In most tertiary general hospitals and academic centres, pulmonary procedures are done in an endoscopy suite that can be shared by gastroenterologists, surgeons, and pulmonologists. In the setting of intensification of the demand for the pulmonary procedures due to expansion in the field of bronchoscopy (EBUS-TBNA, Medical thoracoscopy, Bronchial thermoplasty, bronchoscopic lung volume reduction, low does lung cancer screening, narrow band imaging, Confocal microscopy etc.) and without expansion of endoscopy resources, improvement in the efficiency is the only feasible way to reduce the procedure wait times, length of stays, and costs.

The limitations of the study are that it's a single centre study with small numbers. Another limitation may the introduction of bias when examining the slides of 2 and 16 revolutions. There was no blinding of the pathologist among the slides of 2 and 16 revolutions. The pathologists were not blinded to the suspected diagnosis as well. As a routine, we provide a brief summary of the case on the pathology request forms and also indicate what we want the pathologist to specifically look for. However, the strengths of the study are that it helps to provide decision guiding tool in an area that lacks adequate evidence to-date and provides food for thought for performing a larger randomized double blind control trial to clarify this aspect of EBUSTBNA.

Although EBUS-TBNA is safe, but increasing number of revolutions, conceivably, do increase the chances of inadvertent puncture of the surrounding structures. Additionally, esp. for centers where EBUS is done under conscious sedation, early completion of the procedure is more desirable as it helps to minimize the need for additional sedation, and patient discomfort or struggle associated with the assisting staff holding the patient while bronchoscopist completes the sampling.

In conclusion, more than two revolutions, or "worm like" material on aspirate does not add value in diagnosing malignancy via EBUSTBNA. In patients with high pre-test probability of lung cancer, the current practice of 10-15 revolutions may be unnecessary and demonstration of the adequacy of two revolutions may confer greater safety and efficiency to the procedure. However, in case of high suspicion of a benign disease, it may be preferable to seek worm like material to aid in the establishment of diagnosis.

\section{Conflict of Interest Statement}

A.V., J.A., P.W.T, K.M., and E.S.E have no potential conflicts of interest to report.

\section{References}

1. Ikeda S, Yanai N, Ishikawa S (1968) Flexible bronchofiberscope. Keio J Med 17: 1-16.

2. Navani N, Nankivell M, Woolhouse I, Harrison RN, Munavvar M, et al. (2011) Endobronchial ultrasound-guided transbronchial needle aspiration for the diagnosis of intrathoracic lymphadenopathy in patients with extrathoracic malignancy: a multicenter study. J Thorac Oncol 6: 1505-1509.

3. Gu P, Zhao YZ, Jiang LY, Zhang W, Xin Y, et al. (2009) Endobronchial ultrasound-guided transbronchial needle aspiration for staging of lung cancer: a systematic review and meta-analysis. Eur J Cancer 45: 1389-1396.

4. Tournoy KG, Govaerts E, Malfait T, Dooms C (2011) Endobronchia ultrasound-guided transbronchial needle biopsy for M1 staging of extrathoracic malignancies. Ann Oncol 22: 127-131. 
Citation: Verma A, Abisheganaden J, Teng PW, Mancer K, Edell ES (2015) EBUS-TBNA: Are Two Needle Revolutions (Back and Forth Movement of the Needle Inside the Lymph Node) Adequate for Diagnosis of Lung Cancer?. J Pulm Respir Med 5: 239. doi:10.4172/2161-105X.1000239

5. Navani N, Brown JM, Nankivell M, Woolhouse I, Harrison RN, et al. (2012) Suitability of Endobronchial Ultrasound-guided transbronchial needle aspiration specimens for sub typing and genotyping of non-small cell lung cancer: a multicenter study of 774 patients. Am J Respir Crit Care Med 185: 1316-1322.

6. Puli SR, Reddy JBK, Bechtold ML (2008) "Endoscopic ultrasound: it's accuracy in evaluating mediastinal lymphadenopathy? A meta-analysis and systematic review," World Journal of Gastroenterology 14: 3028-3037,

7. Creemers K, van der Heiden O, Los J, van Esser J, Newhall D, et al. (2011) Endoscopic ultrasound fine needle aspiration in the diagnosis of lymphoma. $J$ Oncol 2011: 785425

8. Lee HS, Lee GK, Lee HS, Kim MS, Lee JM, et al. (2008) Real-time endobronchia ultrasound-guided transbronchial needle aspiration in mediastinal staging of non-small cell lung cancer: how many aspirations per target lymph node station? Chest 134: $368-374$

9. Yarmus LB, Akulian J, Lechtzin N, Yasin F, Kamdar B, et al. (2013) Comparison of 21-gauge and 22-gauge aspiration needle in endobronchial ultrasoundguided transbronchial needle aspiration: results of the American College of Chest Physicians Quality Improvement Registry, Education, and Evaluation Registry. Chest 143: 1036-1043.

10. Nakajima T, Yasufuku K, Takahashi R, Shingyoji M, Hirata T, et al. (2011) Comparison of 21-gauge and 22-gauge aspiration needle during endobronchial ultrasound-guided transbronchial needle aspiration. Respirology 16: 90-94.

11. Shiroyama T, Okamoto N, Suzuki H, Tamiya M, Yamadori T, et al. (2013) Usefulness of High Suction Pressure for Sufficient Tissue Collection during Endobronchial Ultrasound Guided Transbronchial Needle Aspiration. PLOS ONE 8: 1-6.

12. Casal RF, Staerkel GA, Ost D, Almeida FA, Uzbeck MH, et al. (2012) Randomized clinical trial of endobronchial ultrasound needle biopsy with and without aspiration. Chest 142: 568-573.
13. Yarmus L1, Akulian J, Gilbert C, Feller-Kopman D, Lee HJ (2013) Optimizing Endobronchial Ultrasound for Molecular Analysis. How Many Passes Are Needed? Ann Am Thorac Soc 10: 636-643.

14. Diacon AH, Schuurmans MM, Theron J, Brundyn K, Louw M, et al. (2007) Transbronchial needle aspirates: how many passes per target site? Eur Respir J 29: 112-116.

15. Verma A, Jeon K, Koh WJ, Suh GY, Chung MP, et al. (2013) Endobronchial ultrasound-guided transbronchial needle aspiration for the diagnosis of central lung parenchymal lesions. Yonsei Med J 54: 672-678.

16. Alsharif M, Andrade RS, Groth SS, Stelow EB, Pambuccian SE (2008) Endobronchial ultrasound-guided transbronchial fine-needle aspiration: the University of Minnesota experience, with emphasis on usefulness, adequacy assessment, and diagnostic difficulties. Am J Clin Pathol 130: 434-443.

17. Murgu S, Colt S (2011) The Essential EBUS Brochoscopist. Learning bronchoscopy in the world today.

18. Nakajima T, Yasufuku K (2011) The Techniques of Endobronchial UltrasoundGuided Transbronchial Needle Aspiration. Innovations 6: 57-64.

19. Cetinkaya E, Yildiz P, Altin S, Yilmaz V (2004) Diagnostic Value of Transbronchial Needle Aspiration by Wang 22-Gauge Cytology Needle in Intrathoracic Lymphadenopathy. Chest. 125: 527-531.

20. Abu-Hijleh M, El-Sameed Y, Eldridge K, Vadia E, Chiu H, et al. (2013) Linea probe endobronchial ultrasound bronchoscopy with guided transbronchial needle aspiration (EBUS-TBNA) in the evaluation of mediastinal and hila pathology: introducing the procedure to a teaching institution. Lung 191: 109-115.

21. Verma A, Lee MY, Wang C, Hussein NB, Selvi K, et al. (2014) Efficiency of Performing Pulmonary Procedures in a shared Endoscopy Unit: procedure times, turnaround times, delays, and procedure waiting times. Journal of Bronchology and Interventional Pulmonology. 